$1-1-2012$

\title{
Classical Confinement And Outward Convection Of Impurity Ions In The Mst Rfp
}

\author{
S. T.A. Kumar \\ D. J. Den Hartog \\ V.V.Mirnov \\ R. M. Magee
}

Follow this and additional works at: https://researchrepository.wvu.edu/faculty_publications

\section{Digital Commons Citation}

Kumar, S. T. A.; Den Hartog, D. J.; Mirnov, V. V.; and Magee, R. M., "Classical Confinement And Outward Convection Of Impurity Ions In The Mst Rfp" (2012). Faculty Scholarship. 1034.

https://researchrepository.wvu.edu/faculty_publications/1034 


\section{saisar

\section{Classical confinement and outward convection of impurity ions in the MST RFP}

S. T. A. Kumar, D. J. Den Hartog, V. V. Mirnov, K. J. Caspary, R. M. Magee et al.

Citation: Phys. Plasmas 19, 056121 (2012); doi: 10.1063/1.4718310

View online: http://dx.doi.org/10.1063/1.4718310

View Table of Contents: http://pop.aip.org/resource/1/PHPAEN/v19/i5

Published by the American Institute of Physics.

\section{Additional information on Phys. Plasmas}

Journal Homepage: http://pop.aip.org/

Journal Information: http://pop.aip.org/about/about_the_journal

Top downloads: http://pop.aip.org/features/most_downloaded

Information for Authors: http://pop.aip.org/authors

\section{ADVERTISEMENT}

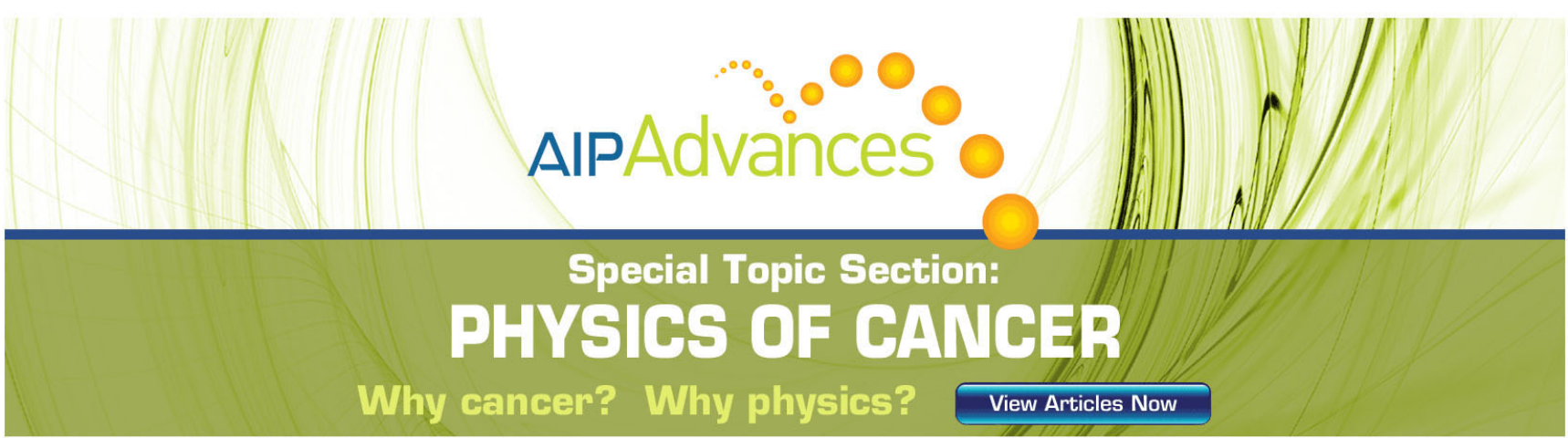




\title{
Classical confinement and outward convection of impurity ions in the MST RFP ${ }^{\text {a) }}$
}

\author{
S. T. A. Kumar, ${ }^{1,2, b)}$ D. J. Den Hartog, ${ }^{1,2}$ V. V. Mirnov, ${ }^{1,2}$ K. J. Caspary ${ }^{1}{ }^{1}$ R. M. Magee, ${ }^{3}$ \\ D. L. Brower, ${ }^{4}$ B. E. Chapman, ${ }^{1}$ D. Craig, ${ }^{5}$ W. X. Ding, ${ }^{4}$ S. Eilerman, ${ }^{1,2}$ \\ G. Fiksel, ${ }^{2,6}$ L. Lin, ${ }^{4}$ M. Nornberg, ${ }^{1,2}$ E. Parke, ${ }^{1}$ J. A. Reusch, ${ }^{1,2}$ and J. S. Sarff ${ }^{1,2}$ \\ ${ }^{1}$ Department of Physics, University of Wisconsin-Madison, Madison, Wisconsin 53706, USA \\ ${ }^{2}$ Center for Magnetic Self-Organization in Laboratory and Astrophysical Plasmas, \\ University of Wisconsin-Madison, Madison, Wisconsin 53706, USA \\ ${ }^{3}$ Department of Physics, West Virginia University, Morgantown, WV 26506, USA \\ ${ }^{4}$ Department of Physics and Astronomy, University of California, Los Angeles, California 90095, USA \\ ${ }^{5}$ Physics Department, Wheaton College, Wheaton, Illinois 60187, USA \\ ${ }^{6}$ Laboratory for Laser Energetics, University of Rochester, Rochester, New York, USA
}

(Received 5 December 2011; accepted 13 April 2012; published online 18 May 2012)

\begin{abstract}
Impurity ion dynamics measured with simultaneously high spatial and temporal resolution reveal classical ion transport in the reversed-field pinch. The boron, carbon, oxygen, and aluminum impurity ion density profiles are obtained in the Madison Symmetric Torus [R. N. Dexter et al., Fusion Technol. 19, 131 (1991)] using a fast, active charge-exchange-recombination-spectroscopy diagnostic. Measurements are made during improved-confinement plasmas obtained using inductive control of tearing instability to mitigate stochastic transport. At the onset of the transition to improved confinement, the impurity ion density profile becomes hollow, with a slow decay in the core region concurrent with an increase in the outer region, implying an outward convection of impurities. Impurity transport from Coulomb collisions in the reversed-field pinch is classical for all collisionality regimes, and analysis shows that the observed hollow profile and outward convection can be explained by the classical temperature screening mechanism. The profile agrees well with classical expectations. Experiments performed with impurity pellet injection provide further evidence for classical impurity ion confinement. (C) 2012 American Institute of Physics. [http://dx.doi.org/10.1063/1.4718310]
\end{abstract}

\section{INTRODUCTION}

The reversed-field pinch (RFP) is an axisymmetric toroidal magnetically confined plasma characterized by low confining magnetic fields and a low safety factor. Unlike tokamaks and stellarators, the toroidal and poloidal fields in the RFP are comparable in magnitude, and the toroidal field reverses direction in the outer region of the plasma (Fig. 1). This results in a reduced field-line connection length (the length along a magnetic field line connecting the toroidally inboard and outboard regions of a magnetic flux surface) and a reduced radial width of the banana orbit (the width of the banana-shaped path traced out by magnetic-mirror-trapped particles in a toroidal plasma). ${ }^{1}$ The banana orbit width is less than the ion gyro radius. As the magnetic fields in the RFP are mostly generated by the plasma itself, $|\mathbf{B}|$ peaks on axis, and the gradient of $|\mathbf{B}|$ is mostly normal to the magnetic flux surfaces, pointing toward the magnetic axis. The $\nabla B \times B$ drift of particles is therefore mostly aligned to the magnetic flux surface. A unique feature resulting from these factors is that the toroidal enhancement of cross-field particle diffusion (neoclassical diffusion) is weak in RFPs, ${ }^{2-4}$ even though some toroidal effects persist, such as enhancement of parallel electrical resistivity due to mirror trapped particles. ${ }^{5,6}$ How-

\footnotetext{
a) Paper NI2 4, Bull. Am. Phys. Soc. 56, 183 (2011).

${ }^{\mathrm{b}}$ Invited speaker. Electronic mail: stkumar@wisc.edu.
}

ever, large amplitude magnetic fluctuations and associated field-line stochasticity in RFPs have historically caused transport much larger than the classical expectation, thus masking classical transport in experiments. In recent years, suppression of magnetic fluctuations has significantly improved RFP performance, ${ }^{7-10}$ exposing the classical nature of transport.

This paper reports an experimental observation of classical impurity ion confinement in the RFP when magnetic fluctuations are suppressed. Impurity ions are expelled from the core until outward convection balances inward diffusion, resulting in a stationary radial impurity density profile that is hollow. The observed impurity density evolution can be explained by the classical temperature screening mechanism in collisional transport theory. Experiments using injection of frozen methane pellets corroborate the observation of classical impurity ion transport. The observation of temperature screening and a hollow impurity profile in the RFP is in contrast to the typical scenario in high-toroidal-field devices (tokamaks and stellarators), where neo-classical transport generally leads to impurity accumulation in the core plasma, ${ }^{11,12}$ even though hollow impurity density profiles due to temperature screening have been observed under certain experimental conditions. ${ }^{13,14}$ Outward convection and a hollow radial profile of impurity ions have also been reported in the multiple helicity and quasi-single-helicity plasma regimes of the RFX-mod reversed-field pinch. ${ }^{15}$ The 


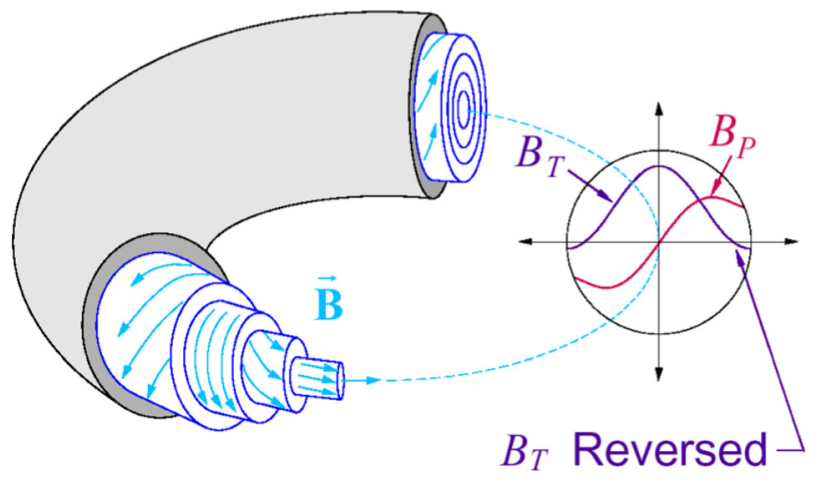

FIG. 1. Schematic diagram of the reversed-field pinch magnetic geometry.

estimated transport coefficients in those experiments are orders of magnitude higher than neoclassical or stochastic expectations. The exact transport mechanism was not determined.

\section{EXPERIMENTAL SETUP}

Experiments are carried out in the Madison Symmetric Torus (MST), which is a large, moderate current $(\leq 600 \mathrm{kA})$ $\mathrm{RFP}$ device with major radius $\mathrm{R}=1.5 \mathrm{~m}$, and minor radius $\mathrm{a}=0.52 \mathrm{~m} .{ }^{16}$ The MST vacuum vessel is aluminum, with a thickness of $5 \mathrm{~cm}$. Partially covering the inner surface of the vessel are tiles and limiters made of graphite, boron nitride, and ceramic, which interact with the plasma during a discharge and become a source of impurities. The identified dominant impurities in MST plasmas are carbon, oxygen, boron, and aluminum. The charge state distribution of these impurities in the plasma depends mainly on the electron temperature (ionization) and the hydrogen neutral density (charge exchange loss), which are significantly different in various plasma regimes in MST.

The typical safety factor $(q)$ profile from a MST standard plasma is shown in Fig. 2(a). Major rational values are marked. The $q$ profile accommodates a large number of rational flux surfaces where tearing modes grow. These multiple coupled tearing modes result in quasi-periodic sawtooth (global magnetic reconnection) events as shown in Fig. 2(b). Magnetic field lines have a high degree of stochasticity both during and between these events. Transport is therefore predominantly stochastic. The global thermal diffusivity $\left(\chi_{E}\right)$ and energy confinement time $\left(\tau_{E}\right)$ are $\chi_{E} \sim 60 \mathrm{~m}^{2} / \mathrm{s}$ and $\tau_{E} \sim 1 \mathrm{~ms}^{17}$

Tearing mode suppression and an associated confinement improvement have been achieved in MST in recent years using "pulsed poloidal current drive" (PPCD), an inductive control technique that reduces the free energy in the current profile that drives tearing instability. PPCD drives a poloidal current at the edge of the plasma by pulsing the toroidal magnetic field. The main characteristics of PPCD discharges are low levels of magnetic fluctuations, higher core electron temperature (see Fig. 3), and improved energy confinement. ${ }^{8-10}$ The estimated energy confinement time $\left(\tau_{E} \sim 12 \mathrm{~ms}\right)$ is increased more than tenfold compared to standard discharges, and the corresponding global thermal diffusivity drops to $\chi_{E} \sim 5 \mathrm{~m}^{2} / \mathrm{s}$. An estimate of the ion thermal conductivity due
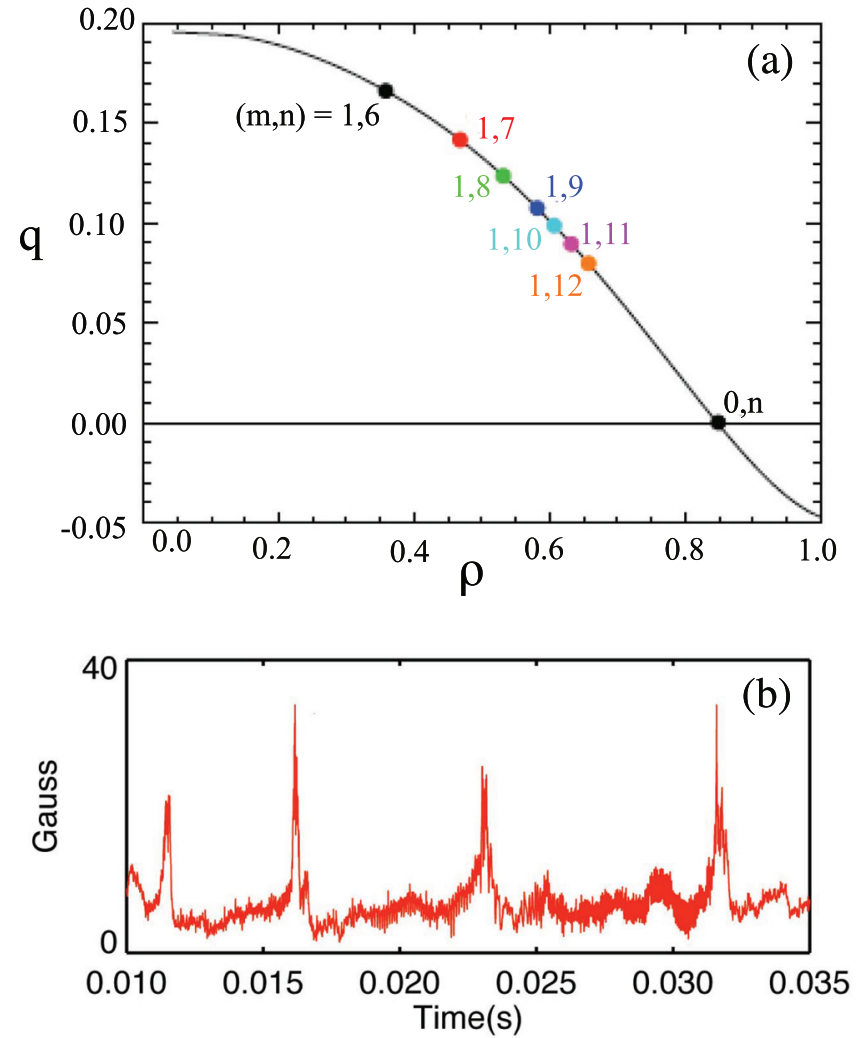

FIG. 2. (a) Typical safety factor profile for standard RFP discharge. Major rational values are marked. (b) Mean amplitude of core-resonant magnetic fluctuations $\left(\sqrt{\sum_{m, n} \tilde{B}_{\theta}^{2}}, \mathrm{~m}=1, \mathrm{n}=7-12\right.$ modes$)$ in a standard RFP discharge exhibiting quasi-periodic sawtooth activity.

to stochasticity $\left(\chi_{i}^{s t}\right)$ can be obtained from $\chi_{E}$, assuming it as the upper bound of stochastic electron conductivity $\left(\chi_{e}^{s t}\right)$, using the relation $\chi_{i}^{s t}=\sqrt{\left(m_{e} T_{i} / m_{i} T_{e}\right)} \chi_{e}^{s t}$, where $m_{i}, T_{i}, m_{e}$, and $T_{e}$ are the ion mass, ion temperature, electron mass, and electron temperature, respectively. ${ }^{17}$ For PPCD conditions, $\chi_{i}^{s t} \lesssim \rho_{i}^{2} \nu_{i i}$, where $\rho_{i}$ is the ion gyro radius ( $\sim 5 \mathrm{~mm}$ for deuterons), and $\nu_{i i}$ is ion-ion collision frequency ( $\sim 2.7 \times 10^{3} / \mathrm{s}$ for deuteron). So the stochastic transport is reduced to below classical level with PPCD, making such discharges good candidates for observing classical ion transport.

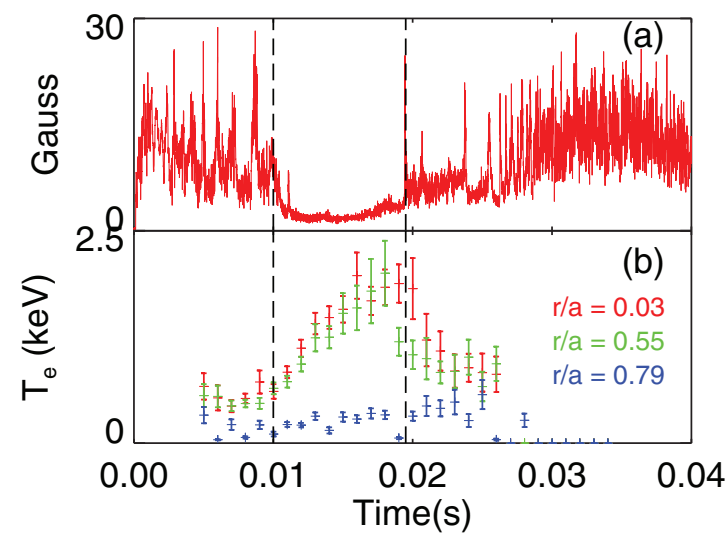

FIG. 3. (a) Mean amplitude of core-resonant tearing modes $\left(\sqrt{\sum_{m, n} \tilde{B}_{\theta}^{2}}\right.$, $\mathrm{m}=1, \mathrm{n}=7-12$ modes) in PPCD discharge. The region between dotted lines represent duration PPCD. (b) Electron temperature measured using a Thomson scattering diagnostic at three different radial locations. 
Experiments reported in this paper are conducted in deuterium PPCD discharges of toroidal plasma current $\sim 400-550 \mathrm{kA}$, line-averaged electron density $n_{e} \sim 1 \times 10^{19} \mathrm{~m}^{-3}$, and electron temperature $\sim 1-2 \mathrm{keV}$. The discharges examined here are all toroidally axisymmetric, unlike some plasmas without PPCD that exhibit a very large core-resonant mode and a nonaxisymmetric helical equilibrium in the plasma core. ${ }^{18,19}$ Impurity density measurements are made using charge-exchange recombination spectroscopy (CHERS). As applied on MST, a beam of neutral hydrogen atoms is injected radially into the plasma, and these atoms charge exchange with the impurity ions in the plasma. The impurity ions are left in an excited lower charge state which then radiatively decays. The Doppler shift and Doppler broadening of this spontaneous de-excitation emission are used to calculate flow velocity and temperature of the initial-charge-state impurity ion population. The density of this population is proportional to the area under the line profile. On MST, the CHERS diagnostic has 11 poloidal viewing chords (Fig. 4) sampling emission along the diagnostic neutral beam (nominal parameters: 45-50 keV, 4-5 A, $20 \mathrm{~ms}$ pulse length). Distinct features of CHERS on MST, which is primarily used for $\mathrm{C}^{+6}$ measurements, are good spatial $(\sim 1 \mathrm{~cm})$ and temporal (up to $10 \mu \mathrm{s}$ ) resolution. The signal-to-noise is greatly enhanced by differencing signals from two lines-of-sight on poloidal planes that are toroidally separated by $\sim 4 \mathrm{~cm}$ (see Fig. 4 ), to measure simultaneously both on-beam and off-beam (background) emission. Measurements are made at one radial location, for one impurity species, per discharge. However, due to high reproducibility, compilation of profiles from
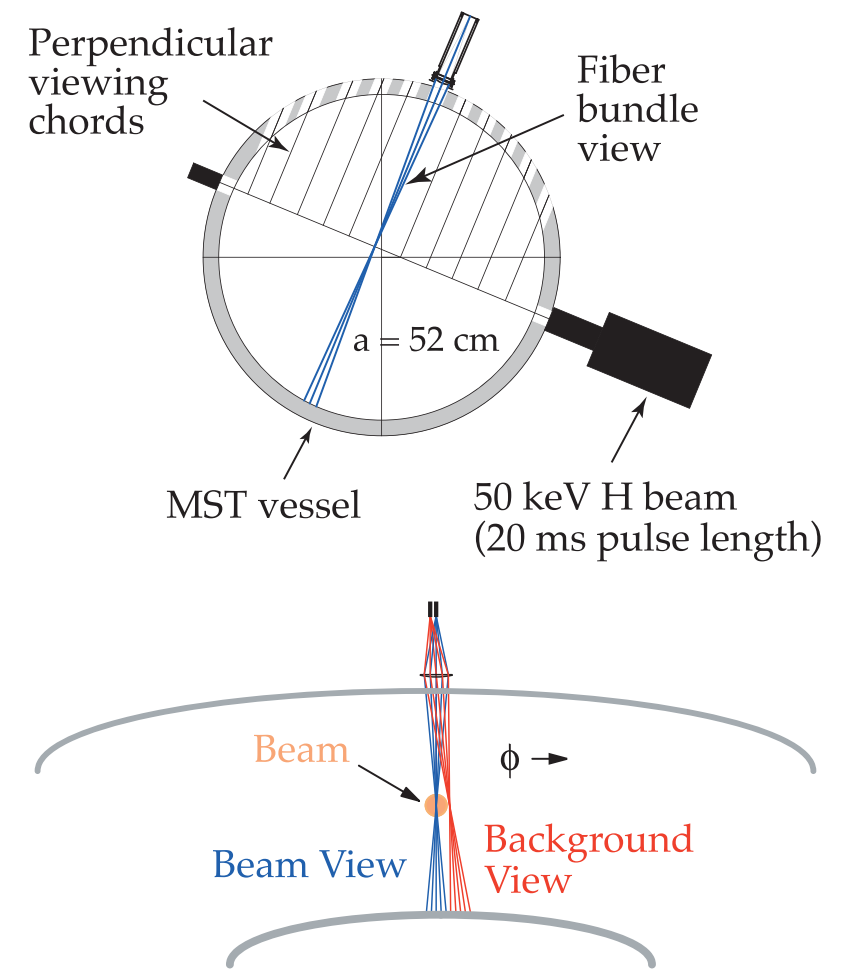

FIG. 4. [Top] Poloidal cross section of MST showing diagnostic neutral beam path and CHERS perpendicular viewing chords. [Bottom] Radial view along the diagnostic neutral beam path showing toroidal displacement of the on-beam and off-beam views. various similar discharges in MST is possible. The custombuilt duo spectrometer ${ }^{20}$ used for the measurements has been calibrated for radiant sensitivity for absolute impurity density calculations from charge exchange emission brightness. Details of the diagnostic setup and the data-fitting model are given elsewhere. ${ }^{20-22}$ For the experiment reported in this paper, measurements are made for $C^{+6}(\mathrm{n}=7-6$ transition, $343.4 \mathrm{~nm}), B^{+5}(\mathrm{n}=6-5$ transition, $298.1 \mathrm{~nm}), O^{+8}(\mathrm{n}=8-7$ transition, $297.5 \mathrm{~nm}), \mathrm{Al}^{+11}(\mathrm{n}=10-9$ transition, $321.05 \mathrm{~nm})$ and $\mathrm{Al}^{+13}(\mathrm{n}=11-10$ transition, $310.65 \mathrm{~nm})$.

\section{EXPERIMENTAL RESULTS}

The temporal behavior of the $C^{+6}$ density and temperature at three minor radial locations $(\rho=r / a \sim 0.02,0.55$, and 0.76 ) are plotted in Figs. 5(a) and 5(b), respectively. A slow decay of the core impurity density after the transition to improved confinement is apparent. The impurity density in the outer region of the plasma, on the other hand, is slowly increasing. The profile in the core region $(0 \leq \rho \leq 0.6)$ approaches a stationary hollow shape toward the end of PPCD. The $C^{+6}$ ion temperature [Fig. 5(b)] remains constant during PPCD, unlike the electron temperature [Fig. 3(b)], as the characteristic electron-ion energy transfer time (several hundreds of milliseconds) is much longer than the PPCD duration. ${ }^{23}$ The radial profile of $C^{+6}$ density and temperature averaged over $\sim 2 \mathrm{~ms}$ toward the end of PPCD is shown in Figs. 5(c) and 5(d) respectively. A hollow radial profile for $C^{+6}$ density is apparent [Fig. 5(c)] whereas the $C^{+6}$ temperature is peaked in the core of the plasma [Fig. 5(d)].

Calculation of the impurity charge state distribution using ADAS (Ref. 24) (without transport) shows that, in PPCD discharges, due to the high electron temperature, light impurities are mostly fully stripped, and the source (lower charge states) of fully stripped ions is small in most of the plasma volume. This result is supported by the experimental observation of a slow decay in the core impurity density, even though the core electron temperature is increasing

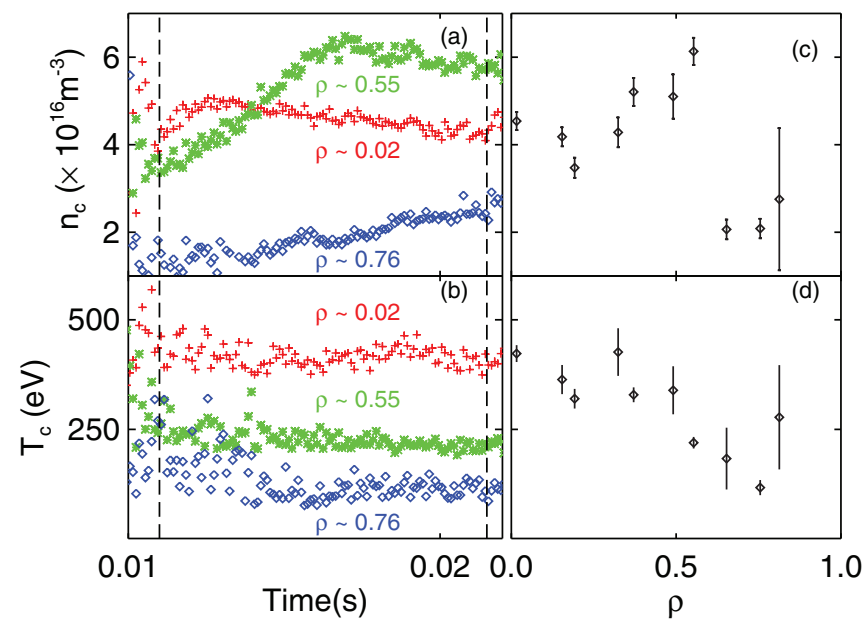

FIG. 5. [Left] Temporal profiles of $C^{+6}$ density (a) and temperature (b) for PPCD discharges at three radial locations: core $(\rho \sim 0.02)$, mid radius $(\rho \sim 0.55)$ and outer region $(\rho \sim 0.76)$. [Right] Radial profiles of $C^{+6}$ density (c) and temperature (d) towards the end of PPCD. Data are averaged over similar discharges. Error bars represent standard deviation of the mean. The region between dotted lines represents duration of good confinement regime. 


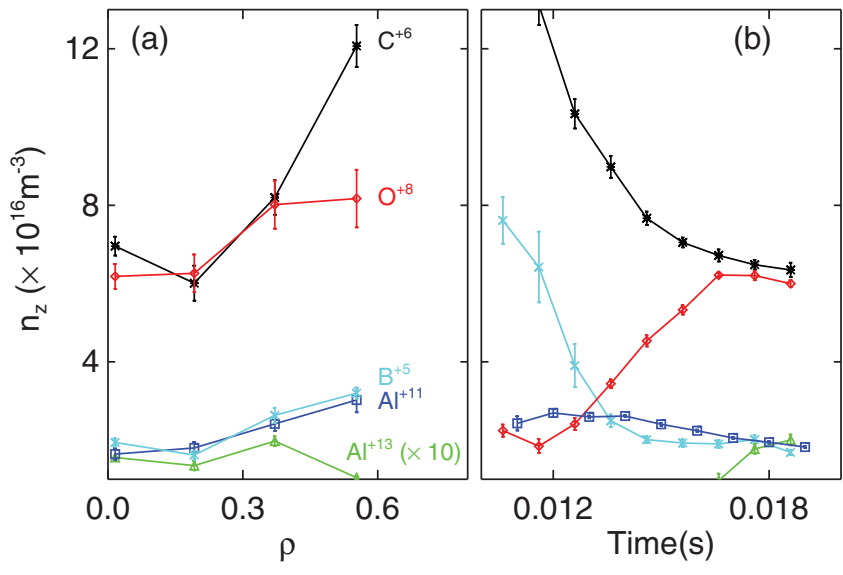

FIG. 6. (a) Radial profiles of various dominant impurity ion densities toward the end of PPCD and (b) on-axis temporal evolution of these impurity densities during PPCD.

during PPCD. The slow decay of the impurity density in the core region concurrent with the increase in impurity density in the outer region hence implies an outward convection of impurities. The slow increase of impurity density in the far outer region $(\rho \geq 0.7)$ is, however, attributed partly to the increasing ionization due to increasing electron temperature.

Density profiles of all identified dominant impurities in MST have been measured (at fewer spatial points) to account for ion-ion collisions in the transport model (described in next section). Figure 6 shows a set of measurements made at a relatively higher plasma current (higher core electron temperature) to access higher charge states of aluminum. ${ }^{25}$ It is apparent that low-Z impurities like boron and carbon have similar features (see also Ref. 26): a clearly hollow profile and slow decay in the core of the plasma. The electron temperature in PPCD is not high enough to fully ionize all the higher-Z impurity atoms in the plasma. Hence, density decay for higher-Z impurities is not expected, even though a hollow radial profile is observed for all impurity species. For example, the $O^{+8}$ density, which has a flat radial profile at the beginning of PPCD, increases due to an increase in ionization and starts to decay only towards the very end of PPCD, slowly evolving to a hollow radial profile [Fig. 6(b)]. Due to the steadily increasing electron temperature, which is not high enough to fully strip all aluminum ions, the aluminum charge state distribution is constantly evolving throughout PPCD. As the aluminum density is an order of magnitude lower than that of carbon, the effect on carbon transport of collisions with aluminum is weak. Densities of aluminum and other impurities are, nevertheless, included in the transport model to account for ion-ion collisions.

\section{MODELING CLASSICAL TRANSPORT}

Since collisional transport of impurities in the RFP is classical for all collisionality regimes, we consider the flux due to classical Coulomb collisions only. The radial impurity flux $\left(\Gamma_{c}\right)$ due to collisions with main ions is given by ${ }^{27}$

$$
\Gamma_{c}=\frac{\nu_{i} \rho_{i}^{2} n_{i}}{2 Z}\left[\frac{\partial \ln P_{i}}{\partial r}-\frac{T_{c} \partial \ln P_{c}}{T_{i} Z \partial r}-\frac{3 \partial \ln T_{i}}{2 \partial r}\right]
$$

for $m_{c} \gg m_{i}$, where subscripts $c$ and $i$ represent impurity and main ions, respectively; $m, P, T, \rho, \nu$, and $Z$ are, respectively, the ion mass, pressure, temperature, gyro radius, collision frequency and, atomic number. The steady-state impurity radial density profile, obtained by setting the radial flux equal to zero, is given by

$$
\frac{n_{c}(r)}{n_{c}(0)}=\left[\frac{n_{i}(r)}{n_{i}(0)}\right]^{Z}\left[\frac{T_{i}(r)}{T_{i}(0)}\right]^{\frac{-Z}{2}-1},
$$

where equilibration of temperatures, $T_{c}=T_{i}$, is assumed. It can be seen from Eq. (2) that the density and temperature gradients of the main ions act in opposite ways: A negative density gradient leads to core impurity accumulation, while a negative temperature gradient leads to impurity expulsion. This is generally called the "temperature screening effect," where the thermal force due to the ion temperature gradient expels impurities from the plasma core. As the ion density profile is nearly flat in the plasma core, and because there exists a strong ion temperature gradient, classical transport analysis predicts a hollow impurity ion density profile for PPCD discharges in MST.

Equation (1) provides the flux of impurity ions due to collisions with the main ions only. Collisions with other impurities could, in principle, significantly modify carbon transport. For example, the mechanism of temperature screening is mass dependent, such that collisions with higher mass impurities could lead to impurity accumulation instead of impurity expulsion. Analysis for multiple impurity ion species yields ${ }^{28}$

$$
\begin{aligned}
\Gamma_{c} & =n_{c} \rho_{c}^{2} \sum_{\beta}\left(\frac{m_{\beta}}{m_{c}+m_{\beta}}\right)^{1 / 2} \nu_{c \beta}\left[-\nabla \ln p_{c}+\frac{Z_{c}}{Z_{\beta}} \nabla \ln p_{\beta}\right. \\
& \left.+\frac{3}{2} \frac{m_{\beta}}{m_{\beta}+m_{c}}\left(1-\frac{m_{c} Z_{c}}{m_{\beta} Z_{\beta}}\right) \nabla \ln T\right],
\end{aligned}
$$

where $\nu_{c \beta}=\frac{16 \pi^{1 / 2}}{2^{3 / 2} 3} n_{\beta} \ln \Lambda\left(\frac{T}{m_{c}}\right)^{1 / 2}\left(\frac{Z_{c} Z_{\beta} e^{2}}{4 \pi \epsilon_{0} T}\right)^{2}$ is the collision frequency of carbon with deuterons and the identified dominant impurity ions (boron, oxygen, and aluminum). All impurities and main ions are assumed to have the same temperature profile, as the impurity-main-ion energy equilibration time $(\sim 0.1 \mathrm{~ms})$ is much less than the impurity life-time ( $\sim 30-40 \mathrm{~ms}$ ). This is consistent with majority and minority ion temperature measurements in PPCD plasmas to date. ${ }^{17}$

The spatial and temporal evolution of the carbon density is modeled for a given initial radial profile using the diffusion equations, $\frac{\partial n_{c}}{\partial t}=-\frac{1}{r} \frac{\partial\left(r \Gamma_{c}\right)}{\partial r}+S$, and $\Gamma_{c}=-D(r) \frac{\partial n_{c}}{\partial r}+V(r) n_{c}$, where $S$ is the source term which is assumed to be zero for the reasons discussed above, $D$ is the classical diffusion coefficient, and $V$ is the convection velocity. The ion temperature and main ion density profiles (calculated from the electron and impurity density profiles assuming charge neutrality) are shown in Figs. 7(a) and 7(b), respectively. Radial profiles of the transport coefficients, $\mathrm{D}$ and $\mathrm{V}$, from the model are given in Figs. 8(a) and 8(b), respectively. It can be seen that the convection velocity is positive (outward) in the core region and reverses its direction at $\rho \sim 0.5$. These opposite directed 

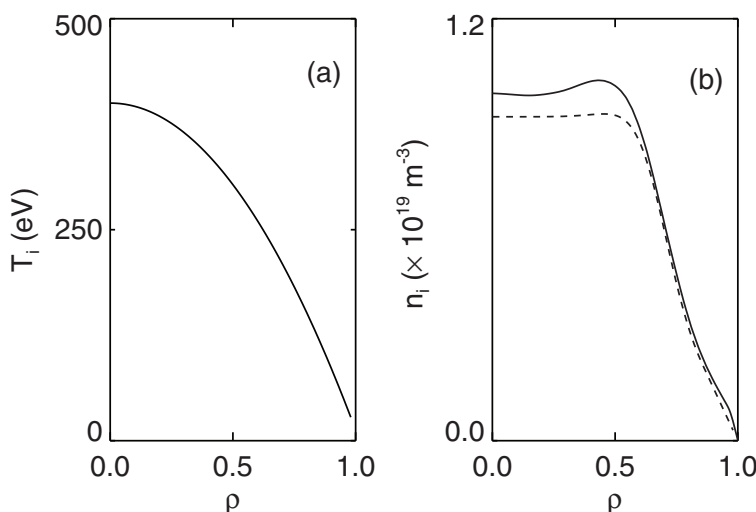

FIG. 7. (a) Radial profile of the ion temperature profile used in the model, (b) radial profile of electron density $n_{e}$ measured by FIR interferometry (solid line) and the radial profile of the main ion density $\left(n_{i}\right)$ used in the model (dotted line), calculated from $n_{e}$ and impurity density profiles, assuming charge neutrality.

velocities cause carbon to "pinch" around $\rho \sim 0.5-0.6$. The model predicts that the profile of carbon evolves to a stationary hollow profile in about $30-40 \mathrm{~ms}$, as shown in Fig. 9(a). The model results agree well with the experimental values both in magnitude and in shape [Fig. 9(b)]. The predicted evolution is also close to the experimental observation, most notably the relatively rapid formation of the hollow shape due to temperature screening.

Temperature screening and hollow impurity ion profiles have been observed in tokamak and stellarator plasmas for particular thermal pressure gradients and collisionality regimes. ${ }^{13,14}$ The temperature screening effect originating from neoclassical physics is very sensitive to the plasma parameters. For example, the observation of temperature screening and a hollow carbon profile in the DIII-D tokamak required both the main and impurity ions to be in the long-mean-free-path regime. ${ }^{13}$ In the Large Helical Device

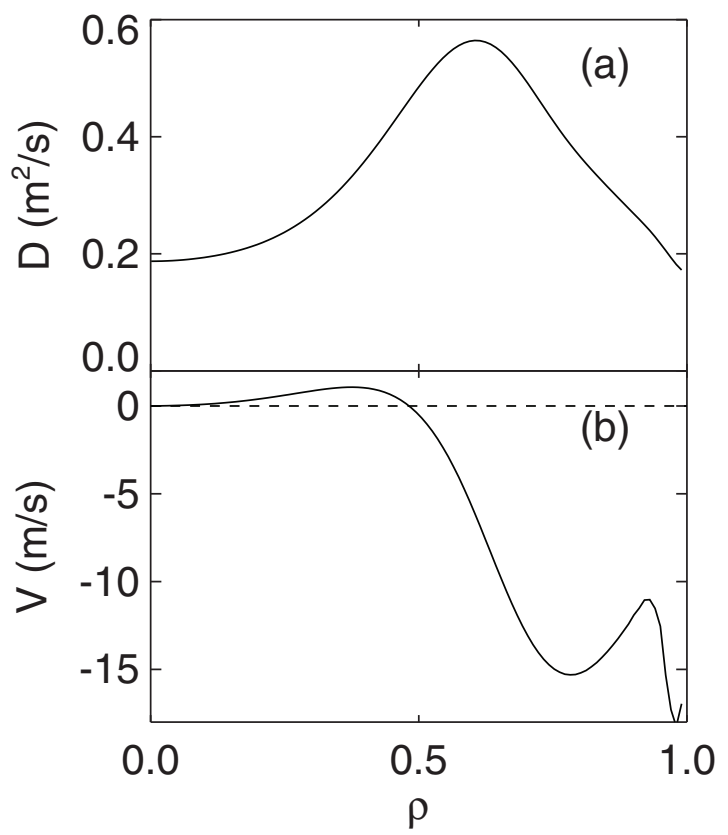

FIG. 8. Radial profiles of the transport coefficients from the classical model: (a) the diffusion coefficient and (b) the convection velocity.

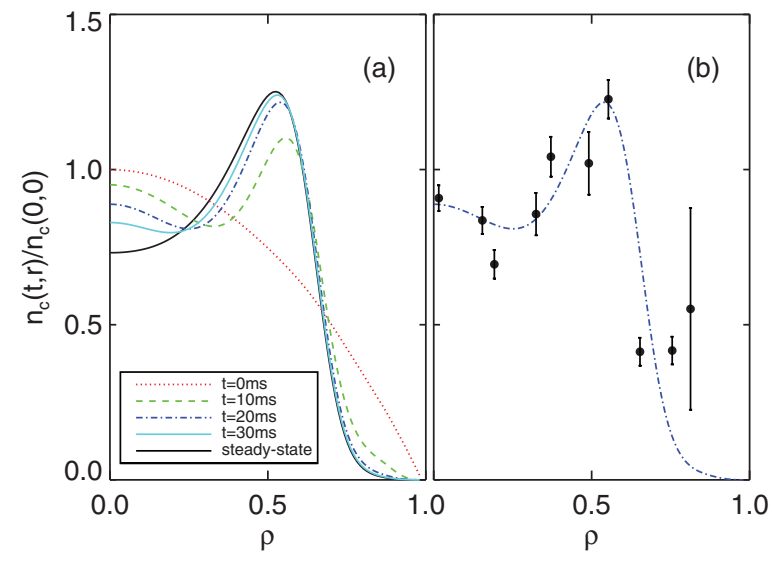

FIG. 9. (a) Time evolution of carbon density profile predicted by the classical model including multiple ion species. Initial parabolic profile and profiles at 10,20 , and $30 \mathrm{~ms}$ are plotted along with the steady-state profile. (b) Model prediction at $20 \mathrm{~ms}$ overlayed on the experimental $\mathrm{C}^{+6}$ density profile (closed circles, scaled for best fit).

stellarator, an "impurity hole" has been observed after carbon pellet injection. This is attributed to the impurity expulsion due to the ion temperature gradient but is inconsistent with the neoclassical predictions. ${ }^{14}$ In contrast to tokamaks and stellarators, hollow profiles and temperature screening in the RFP are determined by classical transport theory and are not sensitive to the plasma collisionality regime.

In order to compare the RFP classical transport prediction to the neoclassical expectation (for example, in a tokamak), the collisionality of $\mathrm{C}^{+6}$, deuterons, and electrons are calculated as shown in Fig. 10. Note that the definition of collisionality in the figure is $\nu L_{C} / V_{\text {th }}$ (instead of the usual expression $\nu^{*} \epsilon^{3 / 2}$ where $\nu^{*}$ is the effective collision frequency and $\epsilon$ is the inverse aspect ratio ${ }^{13}$ ) where $\nu$ is the total collision frequency $\left(=\sum_{\beta} \nu_{\alpha \beta}\right.$, where $\alpha$ represents electron, deuteron, or carbon, and $\beta$ represents all ion species which contribute to the transport), $L_{C}$ is the magnetic field connection length, and $v_{t h}$ is the thermal velocity. Here, $L_{C}$ is calculated directly. (The magnetic field-line connection length can be written as $L_{C}=\pi r / \sin (\theta)$, where $r$ is the minor radius of the flux surface, $\theta=\arctan \left(B_{P} / B_{T}\right)$ is the angle between the field line and the magnetic axis, and $B_{T}$ and $B_{P}$ are toroidal and poloidal components of the magnetic field, respectively.

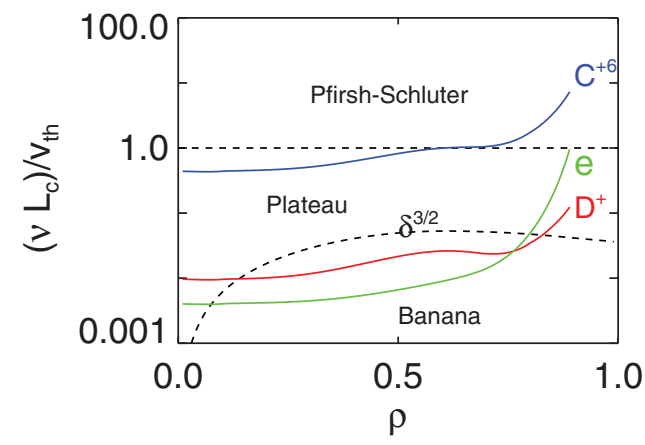

FIG. 10. Collisionality of $\mathrm{C}^{+6}$, deuterons, and electrons for MST parameters. Note that the definition of collisionality is different from the usual expression used for tokamaks and stellarators (see text). 


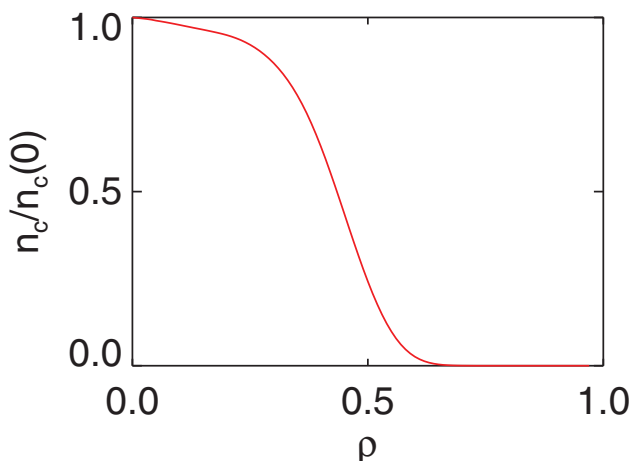

FIG. 11. Equilibrium carbon density profile predicted by neoclassical transport for MST collisionality parameters.

Small-angle approximation for tokamaks and stellarators leads to $L_{C}=\pi r /\left(B_{P} / B_{T}\right)=\pi R q$, where $R$ is the major radius of the device and $q=r B_{T} / R B_{P}$ is the safety factor. Such an approximation is inappropriate for the RFP, especially at $q=0$ surface where $L_{C}=\pi r$, not zero.) and the parameter $\delta$, which defines the boundary between the "banana" and the "plateau" regimes (Fig. 10), takes into account $B_{p} \sim B_{T}$, as appropriate for the RFP. ${ }^{4}\left(\delta=\frac{\left(\Lambda B_{p}^{2}+\epsilon B_{T}^{2}\right)}{\left(B_{p}^{2}+B_{T}^{2}\right)}\right.$ where $\Lambda$ is an asymmetric parameter which depends on plasma pressure and $B_{p}$, and $\epsilon$ is the inverse aspect ratio.) As shown in Fig. $10, C^{+6}$ is in the "plateau regime" of collisionality for most of the plasma volume. Therefore, if plasma with MST collisionality parameters were confined in a tokamak, the $\mathrm{C}^{+6}$ transport would take place in the "plateau regime" of collisionality where the neoclassical theory predicts an equilibrium profile of the form ${ }^{29} \frac{n_{c}(r)}{n_{c}(0)}=\left[\frac{n_{i}(r)}{n_{i}(0)}\right]^{Z}\left[\frac{T_{i}(r)}{T_{i}(0)}\right]^{1.5 Z}$. Note that the temperature gradient has a positive exponent in this case, leading to impurity accumulation in the core instead of expulsion, resulting in a core-peaked impurity profile as shown in Fig. 11.

\section{IMPURITY PELLET INJECTION}

Experiments performed with impurity pellet injection provide further evidence for classical impurity ion confinement when tearing instabilities are suppressed. Frozen methane $\left(\mathrm{CH}_{4}\right)$ pellets of diameter $\sim 1 \mathrm{~mm}$ and length $\sim 2 \mathrm{~mm}$ are injected into the core of PPCD plasmas with a speed of $\sim 200 \mathrm{~m} / \mathrm{s}$ using MST's pellet injector. ${ }^{30}$ This is the first time that the pellet injector, which is designed for frozen deuterium pellets, has been used for injecting frozen methane pellets. As methane has a significantly higher triple point than deuterium, some modifications to the injector operations were required.

The time evolution of the on-axis $\mathrm{C}^{+6}$ density for a pellet-injected PPCD discharge is shown in Fig. 12(a). The characteristic decay time $\left(\tau_{c}\right)$ is obtained from an exponential fit to the core $\mathrm{C}^{+6}$ density decay phase. Pellet injection generally results in an increase in the plasma density and a more modest reduction in magnetic fluctuations compared to the no-pellet case. This exposes the sensitivity of $\tau_{c}$ to magnetic fluctuation amplitude (and/or plasma density). It has been observed that the decay time approaches a large value as the magnetic fluctuations are reduced [Fig. 12(b)]. To put
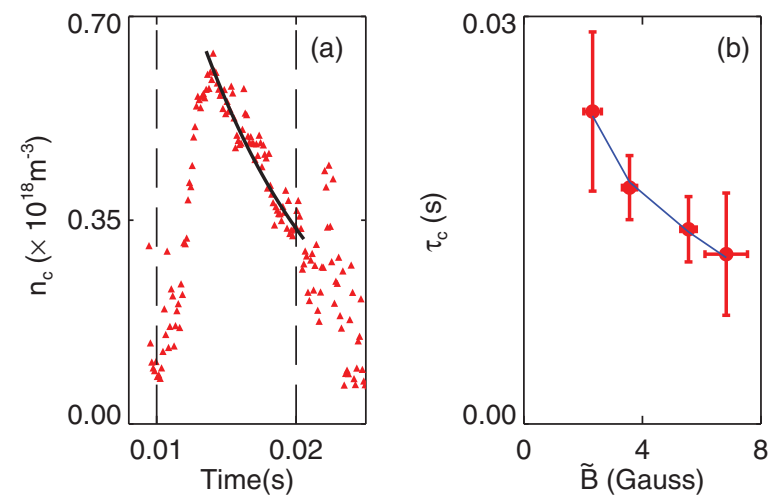

FIG. 12. (a) Time evolution of on-axis $\mathrm{C}^{+6}$ density during a pellet-injected PPCD discharge showing the increase due to pellet injection and a slow decay afterward. Time window between dotted vertical lines represents duration of PPCD. Exponential fit to the decay is also shown. (b) On-axis impurity decay time correlated with the mean amplitude of core-resonant magnetic fluctuations. Solid line shows the empirical relationship, $\tau_{c} \propto \frac{1}{n_{e}^{0.1} \tilde{B}^{0.5}}$ (see text).

this in perspective, the core decay time calculated by the classical model is $\sim 30-45 \mathrm{~ms}$. (This is a rough estimate obtained by a model analogous to the one described by Fig. 9(a). A core-peaked initial carbon profile is used in the model to simulate pellet-injected scenario. Note that, apart from relatively higher $n_{e}$ and lower $T_{e}$, pellet-injected plasmas may exhibit a low ion temperature, higher neutral density, and significantly different higher-Z impurity compositions. As these plasmas are not well diagnosed, $\tau_{c}$ estimated by the model may have large uncertainty.) It has also been observed that $\tau_{c}$ is related to the plasma density $\left(n_{e}\right)$ and magnetic field fluctuation amplitude $(\tilde{B})$ by an empirical relation, $\tau_{c} \propto \frac{1}{\left(n_{e}^{0.1} \tilde{B}^{0.5}\right)}$, for the range of parameters in our experiment [solid line in Fig. 12(b)]. For reference, particle transport in a stochastic magnetic field would be expected to scale as ${ }^{31} \tau_{c} \propto \frac{1}{\tilde{B}^{2}}$, of course applicable only when stochastic transport is important. All cases in Fig. 12(b) have reduced magnetic fluctuations relative to the standard RFP case where stochastic transport is expected to be dominant, so transport that is not fully stochastic might be expected. There may be other implicit dependencies, especially the plasma temperature. Nevertheless, supporting experimental evidence has thus been obtained that the impurity ion confinement approaches classical in MST, when magnetic fluctuations are reduced.

\section{CONCLUSION}

This paper presents experimental evidence for classical ion transport in the reversed-field pinch. When turbulent transport is suppressed, the core $\mathrm{C}^{+6}$ impurity density decays slowly in time, evolving to a hollow radial profile, indicating outward convection of impurity ions. The profiles agree well with expectations from simple classical Coulomb collisions. The impurity expulsion is due to the "temperature screening effect" where the thermal force due to an ion temperature gradient expels impurities from the core of the plasma. Experiments with impurity pellet injection corroborate the observation of classical impurity ion confinement. 


\section{ACKNOWLEDGMENTS}

This work is supported by the U.S. Department of Energy under cooperative agreement DE-FCO2-05ER54814 for MST and by National Science Foundation-PHY0821899.

${ }^{1}$ K. Miyamoto, Fundamentals of Plasma Physics and Controlled Fusion (Iwanami Book Service Center, 1997).

${ }^{2}$ T. S. Chen, A. Nagata, K. Sato, H. Ashida, and T. Amano, J. Phys. Soc. Jpn. 61, 530 (1992).

${ }^{3}$ H. Oshiyama and S. Masamune, J. Phys. Soc. Jpn. 52, 2041 (1983).

${ }^{4}$ M. Wakatani and R. Itatani, J. Phys. Soc. Jpn. 34, 181 (1973).

${ }^{5}$ M. R. Stoneking, J. T. Chapman, D. J. Den Hartog, S. C. Prager, and J. S. Sarff, Phys. Plasmas 5, 1004 (1998).

${ }^{6}$ J. A. Reusch, J. K. Anderson, D. J. Den Hartog, F. Ebrahimi, D. D. Schnack, H. D. Stephens, and C. B. Forest, Phys. Rev. Lett. 107, 155002 (2011).

${ }^{7}$ J. S. Sarff, S. A. Hokin, H. Ji, S. C. Prager, and C. R. Sovinec, Phys. Rev. Lett. 72, 3670 (1994).

${ }^{8}$ B. E. Chapman, A. F. Almagri, J. K. Anderson, T. M. Biewer, P. K. Chattopadhyay, C.-S. Chiang, D. Craig, D. J. Den Hartog, G. Fiksel, C. B. Forest, A. K. Hansen, D. Holly, N. E. Lanier, R. O'Connell, S. C. Prager, J. C. Reardon, J. S. Sarff, M. D. Wyman, D. L. Brower, W. X. Ding, Y. Jiang, S. D. Terry, P. Franz, L. Marrelli, and P. Martin, Phys. Plasmas 9, 2061 (2002).

${ }^{9}$ P. Franz, L. Marrelli, P. Piovesan, B. E. Chapman, P. Martin, I. Predebon, G. Spizzo, R. B. White, and C. Xiao, Phys. Rev. Lett. 92, 125001 (2004).

${ }^{10}$ R. O’Connell, D. J. Den Hartog, C. B. Forest, J. K. Anderson, T. M. Biewer, B. E. Chapman, D. Craig, G. Fiksel, S. C. Prager, J. S. Sarff, S. D. Terry, and R. W. Harvey, Phys. Rev. Lett. 91, 045002 (2003).

${ }^{11}$ R. Dux, C. Giroud, R. Neu, A. G. Peeters, J. Stober, K.-D. Zastrow, and Contributors to the EFDA-JET Workprogramme, J. Nucl. Mater. 313-316, 1150 (2003).

${ }^{12}$ R. Burhenn, Y. Feng, K. Ida, H. Maassberg, K. McCarthy, D. Kalinina, M. Kobayashi, S. Morita, Y. Nakamura, H. Nozato, S. Okamura, S. Sudo, C. Suzuki, N. Tamura, A. Weller, M. Yoshinuma, and B. Zurro, Nucl. Fusion 49, 065005 (2009).

${ }^{13}$ M. R. Wade, W. A. Houlberg, and L. R. Baylor, Phys. Rev. Lett. 84, 282 (2000).

${ }^{14}$ M. Yoshinuma, K. Ida, M. Yokoyama, M. Osakabe, K. Nagaoka, S. Morita, M. Goto, N. Tamura, C. Suzuki, S. Yoshimura, H. Funaba, Y. Takeiri, K. Ikeda, K. Tsumori, O. Kaneko, and the LHD Experimental Group, Nucl. Fusion 49, 062002 (2009).

${ }^{15}$ S. Menmuir, L. Carraro, A. Alfier, F. Bonomo, A. Fassina, G. Spizzo, and N. Vianello, Plasma Phys. Controlled Fusion 52, 095001 (2010).
${ }^{16}$ R. N. Dexter, D. W. Kerst, T. W. Lovell, S. C. Prager, and J. C. Sprott, Fusion Technol. 19, 131 (1991).

${ }^{17}$ J. S. Sarff, J. K. Anderson, T. M. Biewer, D. L. Brower, B. E. Chapman, P. K. Chattopadhyay, D. Craig, B. Deng, D. J. Den Hartog, W. X. Ding, G. Fiksel, C. B. Forest, J. A. Goetz, R. O'Connell, S. C. Prager, and M. A. Thomas, Plasma Phys. Controlled Fusion 45, A457 (2003).

${ }^{18}$ R. Lorenzini, E. Martines, P. Piovesan, D. Terranova, P. Zanca, M. Zuin, A. Alfier, D. Bonfiglio, F. Bonomo, A. Canton, S. Cappello, L. Carraro, R. Cavazzana, D. F. Escande, A. Fassina, P. Franz, M. Gobbin, P. Innocente, L. Marrelli, R. Pasqualotto, M. E. Puiatti, M. Spolaore, M. Valisa, N. Vianello, and P. Martin, Nat. Phys. 5, 570 (2009).

${ }^{19}$ W. F. Bergerson, F. Auriemma, B. E. Chapman, W. X. Ding, P. Zanca, D. L. Brower, P. Innocente, L. Lin, R. Lorenzini, E. Martines, B. Momo, J. S. Sarff, and D. Terranova, Phys. Rev. Lett. 107, 255001 (2011).

${ }^{20}$ D. Craig, D. J. Den Hartog, D. A. Ennis, S. Gangadhara, and D. Holly, Rev. Sci. Instrum. 78, 013103 (2007).

${ }^{21}$ D. J. Den Hartog, D. Craig, D. A. Ennis, G. Fiksel, S. Gangadhara, D. J. Holly, J. C. Reardon, V. I. Davydenko, A. A. Ivanov, A. A. Lizunov, M. G. O’Mullane, and H. P. Summers, Rev. Sci. Instrum. 77, 10F122 (2006).

${ }^{22}$ S. Gangadhara, D. Craig, D. A. Ennis, and D. J. Den Hartog, Rev. Sci. Instrum. 77, 10F109 (2006).

${ }^{23}$ B. Chapman, J. Ahn, A. Almagri, J. Anderson, F. Bonomo, D. Brower, D. Burke, K. Caspary, D. Clayton, S. Combs, W. Cox, D. Craig, B. Deng, D. Den Hartog, W. Ding, F. Ebrahimi, D. Ennis, G. Fiksel, C. Forest, C. Foust, P. Franz, S. Gangadhara, J. Goetz, M. Kaufman, J. Kulpin, A. Kuritsyn, R. Magee, M. Miller, V. Mirnov, P. Nonn, R. O’Connell, S. Oliva, S. Prager, J. Reusch, J. Sarff, H. Stephens, M. Wyman, and T. Yates, Nucl. Fusion 49, 104020 (2009).

${ }^{24}$ H. P. Summers, The Atomic Data and Analysis Structure User Manual, Version 2.6. www.adas.ac.uk (2004).

${ }^{25}$ S. T. A. Kumar, D. J. Den Hartog, B. E. Chapman, M. O’Mullane, M. Nornberg, D. Craig, S. Eilerman, G. Fiksel, E. Parke, and J. Reusch, Plasma Phys. Controlled Fusion 54, 012002 (2012).

${ }^{26}$ S. T. A. Kumar, D. J. Den Hartog, R. M. Magee, G. Fiksel, and D. Craig, Plasma Phys. Controlled Fusion 53, 032001 (2011).

${ }^{27}$ S. P. Hirshman and D. J. Sigmar, Nucl. Fusion 21, 1079 (1981).

${ }^{28}$ V. Zhdanov, Transport Processes in Multicomponent Plasma (Taylor \& Francis, London, 2002).

${ }^{29}$ K. W. Wenzel and D. J. Sigmar, Nucl. Fusion 30, 1117 (1990).

${ }^{30}$ S. K. Combs, L. R. Baylor, D. T. Fehling, P. W. Fisher, C. R. Foust, D. A. Rasmussen, J. B. Wilgen, B. E. Chapman, S. P. Oliva, S. C. Prager, J. S. Sarff, M. D. Wyman, D. L. Brower, W. X. Ding, S. D. Terry, and B. H. Deng, Fusion Sci. Technol. 44, 513 (2003).

${ }^{31}$ A. B. Rechester and M. N. Rosenbluth, Phys. Rev. Lett. 40, 38 (1978). 